\title{
Segmentation of Dynamic PET Images Using Cluster Analysis
}

\author{
Koon-Pong Wong, Member, IEEE, Dagan Feng, Senior Member, IEEE, Steven R. Meikle, Senior Member, IEEE, \\ and Michael J. Fulham
}

\begin{abstract}
Quantitative positron emission tomography (PET) studies provide in vivo measurements of dynamic physiological and biochemical processes in humans. A limitation of PET is an inability to provide precise anatomic localization due to relatively poor spatial resolution when compared to magnetic resonance (MR) imaging. Manual placement of region-of-interest (ROI) is commonly used in clinical and research settings in analysis of PET datasets. However, this approach is operator dependent and time-consuming. A semi- or fully-automated ROI delineation (or segmentation) method offers advantages by reducing operator error/subjectivity and thereby improving reproducibility. In this work, we describe an approach to automatically segment dynamic PET images using cluster analysis and we validate our approach with a simulated phantom study and assess its performance with real dynamic PET data. Our preliminary results suggest that cluster analysis can automatically segment tissues in dynamic PET studies and has the potential to replace manual ROI delineation for some applications.
\end{abstract}

Index Terms-Cluster analysis, functional imaging, positron emission tomography (PET), segmentation.

\section{INTRODUCTION}

$\mathbf{N}$ UCLEAR medicine imaging modalities such as positron emission tomography (PET) and SPECT, can measure functional changes in tissues. In routine dynamic PET studies, region-of-interest (ROI) delineation is required for quantitative analysis [1]. A common approach is to identify the anatomic structures by placing ROIs directly on the PET images, and the underlying tissue time-activity curves (TACs) are then extracted for subsequent analysis. This ROI analysis approach, although widely used in clinical and research settings, is operator dependent and thus prone to reproducibility errors and it is also time consuming. In addition, this approach is problematic when

\footnotetext{
Manuscript received November 3, 2000; revised eptember 1, 2001. This work was supported by the National Health and Medical Research Council (NHMRC) of Australia under Grant 980042.

K.-P. Wong is with the Department of PET and Nuclear Medicine, Royal Prince Alfred Hospital, Camperdown, NSW 2050, Australia. He is also with the Basser Department of Computer Science, The University of Sydney, NSW 2006, Australia (e-mail: kpong@ nucmed.rpa.cs.nsw.gov.au).

D. Feng is with the Basser Department of Computer Science, The University of Sydney, NSW 2006, Australia. He is also with the Department of Electronic and Information Engineering, The Hong Kong Polytechnic University, Hong Kong (e-mail: feng@cs.usyd.edu.au).

S. R. Meikle is with the Department of PET and Nuclear Medicine, Royal Prince Alfred Hospital, Camperdown, NSW 2050, Australia (e-mail: steve@cs.usyd.edu.au).

M. J. Fulham is with the Department of PET and Nuclear Medicine, Royal Prince Alfred Hospital, Camperdown, NSW 2050, Australia. He is also with the Faculty of Medicine, The University of Sydney, NSW 2006, Australia (e-mail: mfulham@med.usyd.edu.au).

Publisher Item Identifier S 0018-9499(02)01626-X.
}

applied to small structures because of the partial volume effects from the low spatial resolution of PET [2]. Unfortunately, many structures of interest, particularly in the brain, are often smaller than the spatial resolution of the PET scanner and corrections aided by anatomical imaging modalities such as computed tomography (CT) and magnetic resonance (MR) are required [3], [4]. A number of registration techniques have been developed for this process [5]. Brain structures can also be identified using a standardized reference coordinate system or PET data can be fitted to a standard anatomical atlas with the aid of anatomical landmarks or contours [6]-[8]. Precise alignment between the anatomic and PET images is necessary for these methods. Importantly, methods that use registration to a standard coordinate system are problematic when patients with pathological processes (e.g., tumors and atrophy) are studied.

Automatic segmentation for three-dimensional (3-D) data, however, is typically not a trivial task. A number of methods for segmentation of MR datasets have been reported [9]-[11]. In MR imaging, it is relatively easy to identify in the brain, grey matter, white matter, cerebrospinal fluid (CSF), and extracranial tissues. But segmentation in PET and SPECT is more difficult because in addition to inherently poor spatial resolution and signal-to-noise ratio (SNR), there is bias due to scatter, signal attenuation, and patient motion during scanning. Notwithstanding these issues, application of automatic segmentation as an alternative to manual ROI delineation has attracted interest recently with the improved spatial resolution of PET and SPECT systems. The advantages of automatic segmentation include more consistent and reproducible results and an overall reduction in time for data analysis.

Cluster analysis is a multivariate data analysis technique that has been used in psychiatry and sociology for many years. One of the major aims of cluster analysis is to partition a large number of objects according to certain criteria into a smaller number of clusters that are mutually exclusive and exhaustive such that the objects within a cluster are similar to each other while objects drawn from different clusters are dissimilar [12]. Cluster analysis has also been applied to neuroimaging to help analyze data obtained from functional MRI (FMRI) [13], [14] and PET [15]-[17]. An example was given by O'Sullivan [15] who demonstrated the use of cluster analysis to partition a set of tissue TACs into a set of homogeneous TACs and it was found that a convex linear combination of those homogeneous TACs using a mixture model can accurately represent the original data while providing quantitative parametric images. It was also demonstrated that an improved SNR can be achieved in applying cluster analysis to dynamic PET data [16], [17]. 
This study is motivated by our on-going work on a noninvasive modeling approach for quantification of $\left[{ }^{18} \mathrm{~F}\right]$ fluorodeoxyglucose (FDG) PET studies where several ROIs of distinct kinetics are required [18]. However, manual delineation of ROIs restrain the reproducibility of the proposed modeling technique. In this study, we describe an approach to automatically segment dynamic PET images using cluster analysis and we validate it using a simulated dynamic 2- $\left[{ }^{11} \mathrm{C}\right]$ thymidine PET study with a slice of the Zubal phantom [19] and assess its clinical potential with dynamic FDG-PET studies.

\section{MATERIALS AND Methods}

\section{A. Segmentation Scheme}

The segmentation method is based on cluster analysis. Our aim is to classify a number of tissue TACs according to their shape and magnitude into a smaller number of distinct characteristic classes that are mutually exclusive so that the tissue TACs within a cluster are similar to one another but are dissimilar to those drawn from other clusters. The clusters (or clustered ROIs) represent the locations in the images where the tissue TACs have similar kinetics. The kinetic curve associated with a cluster (i.e., cluster centroid) is the average of TACs in the cluster. Suppose that there exists $k$ characteristic curves in the dynamic PET data matrix $\boldsymbol{M}$, which has $m$ tissue TACs and $n$ time frames with $k \ll m$ and that any tissue TAC belongs to only one of the $k$ curves. The clustering algorithm then segments the dynamic PET data into $k$ curves automatically based on a weighted least-squares distance measure $\mathcal{D}$, which is defined as

$$
\mathcal{D}\left\{z_{i}, \mu_{j}\right\}=\sum_{j=1}^{k} \sum_{i=1}^{m}\left\|z_{i}-\mu_{j}\right\|_{W}^{2}
$$

where $z_{i} \in \Re^{n}$ is the $i$ th tissue TAC in the data; $\mu_{j} \in \Re^{n}$ is the centroid of cluster $C_{j}$; and $W \in \Re^{n \times n}$ is a square matrix containing the weighting factors on the diagonal and zero for the off-diagonal entries. The weighting factors were used to boost the degree of separation between any TACs that have different uptake patterns but have similar least-squares distances to a given cluster centroid. They were chosen to be proportional to the scanning intervals of the experiment. Although this is not necessarily an optimal weighting, reasonably good clustering results can be achieved.

There is no explicit assumption on the structure of data and the clustering process proceeds automatically in an unsupervised manner. The minimal assumption for the clustering algorithm is that the dynamic PET data can be represented by a finite number of kinetics. As the number of clusters $k$ for a given data set is usually not known a priori, $k$ is usually determined by trial and error. In addition, the initial cluster centroid in each cluster is initialized randomly to ensure that all clusters are nonempty. Each tissue TAC is then allocated to its nearest cluster centroid according to the following criterion:

$$
\begin{array}{r}
\left\|z_{l}-\mu_{i}\right\|_{\boldsymbol{W}}^{2}<\left\|z_{l}-\mu_{j}\right\|_{\boldsymbol{W}}^{2} \Rightarrow z_{l} \in C_{i} \\
\forall i, j=1,2, \ldots, k, \quad i \neq j
\end{array}
$$

where $z_{l} \in \Re^{n}$ is the $l$ th tissue TAC in $\boldsymbol{M} ; \mu_{i} \in \Re^{n}$ and $\mu_{j} \in \Re^{n}$ are the $i$ th and $j$ th cluster centroid, respectively; and $C_{i}$ represents the $i$ th cluster set. The centroids in the clusters are updated based on (2) so that (1) is minimized. The above allocation and updating processes are repeated for all tissue TACs until there is no reduction in moving a tissue TAC from one cluster to another. On convergence, the cluster centroids are mapped back to the original data space for all voxels. An improved SNR can be achieved because each voxel in the mapped data space is represented by one of the cluster centroids each of which possesses a higher statistical significance than an individual TAC.

Convergence to a global minimum is not always guaranteed because the final solution is not known a priori unless certain constraints are imposed on the solution that may not be feasible in practice. In addition, there may be several local minima in the solution space when the number of clusters is large. Restarting the algorithm with different initial cluster centroids is necessary to identify the best possible minimum in the solution space.

The algorithm is similar to the $K$-means type Euclidean clustering algorithm [20]. However, the $K$-means type Euclidean clustering algorithm requires that the data are normalized and it does not guarantee that the within-cluster cost is minimized since no testing is performed to check whether there is any cost reduction if an object is moved from one cluster to another.

The proposed method would work on 3-D datasets as it only makes use of the temporal information rather than the spatial information although it could be if one wanted contiguous regions to be clustered. In this study, however, the clustering was performed independently on each slice.

\section{B. Cluster Validation}

As mentioned earlier, the optimum number of clusters for a given dataset is usually not known a priori. It is advantageous if this number can be determined based on the given dataset. In this study, a model-based approach was adopted to cluster validation based on two information-theoretic criteria, namely, Akaike information criterion (AIC) [21] and Schwarz criterion (SC) [22], assuming that the data can be modeled by an appropriate probability distribution function (e.g., Gaussian). Both criteria determine the optimal model order by penalizing the use of a model that has a greater number of clusters. Thus, the number of clusters that yields the lowest value for AIC and/or SC is selected as the optimum. The use of AIC and SC has some advantages compared to other heuristic approaches such as the "bootstrap" resampling technique, which requires a large amount of stochastic computation. This model-based approach is relatively flexible in evaluating the goodness-of-fit and a change in the probability model of the data does not require any change in the formulation except the modeling assumptions. It is noted, however, that both criteria may not indicate the same model as the optimum [22].

The validity of clusters is also assessed visually and by thresholding the average mean-squared error (MSE) across clusters, which is defined as

$$
\mathrm{MSE}=\frac{1}{k} \sum_{j=1}^{k} \sum_{i=1}^{m}\left\|z_{i}-\mu_{j}\right\|_{W}^{2}
$$




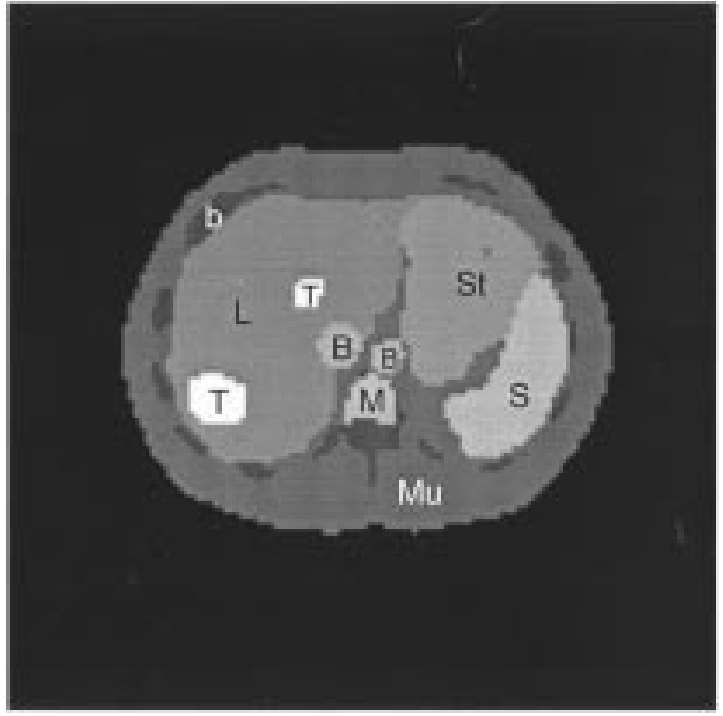

Fig. 1. A slice of the Zubal phantom. $\mathrm{B}=$ blood vessels; $\mathrm{b}=$ bone; $\mathrm{L}=$ liver; $\mathrm{M}=$ marrow; $\mathrm{Mu}=$ muscle; $\mathrm{S}=$ spleen; $\mathrm{St}=$ stomach; $\mathrm{T}=$ tumor.

Both approaches are subjective, but they can provide an insight into the "correct" number of clusters.

\section{Validation Study}

To examine the validity of the segmentation scheme, we simulated a dynamic $2-\left[{ }^{11} \mathrm{C}\right]$ thymidine (a marker of cell proliferation) PET study. The $2-\left[{ }^{11} \mathrm{C}\right]$ thymidine was chosen because it is being increasingly used in the research setting to evaluate cancer and treatment response and it offers theoretical advantages over FDG such as greater specificity in the assessment of malignancy. Also, the kinetics are very similar for most tissues and the data are typically quite noisy. Thus, thymidine data represent a challenging example for testing the clustering algorithm.

Typical $2-\left[{ }^{11} \mathrm{C}\right]$ thymidine kinetics for different tissues were derived from eight patients. The data were acquired on an ECAT 931 scanner (CTI/Siemens, Knoxville, TN). The dynamic PET data were acquired over 60 min with a typical sampling schedule $(10 \times 30 \mathrm{~s}, 5 \times 60 \mathrm{~s}, 5 \times 120 \mathrm{~s}, 5 \times 180 \mathrm{~s}$, $5 \times 300 \mathrm{~s}$ ) and the tracer TAC in blood was measured with a radial artery catheter following tracer administration. Images were reconstructed using filtered backprojection (FBP) with a Hann filter cutoff at the Nyquist frequency. ROIs were drawn over the PET images to obtain tissue TACs in bone, bone marrow, blood pool, liver, skeletal muscle, spleen, stomach, and tumor. Impulse response functions (IRFs) corresponding to these tissues were determined by spectral analysis of the tissue TACs [23]. The average IRFs for each common tissue type were obtained by averaging the spectral coefficients across the subjects and convolved with a typical arterial input function, resulting in typical TACs for each tissue. The TACs were then assigned to the corresponding tissue types in a single slice of the Zubal phantom [19], which included blood vessels, bone, liver, bone marrow, muscle, spleen, stomach, a large and small tumor in the liver (see Fig. 1). A dynamic sequence of sinograms was obtained by forward projecting the images into $3.13 \mathrm{~mm}$ bins on a $192 \times 256$ grid. Attenuation was included in the simulations for the purpose of obtaining the correct scaling of the noise.

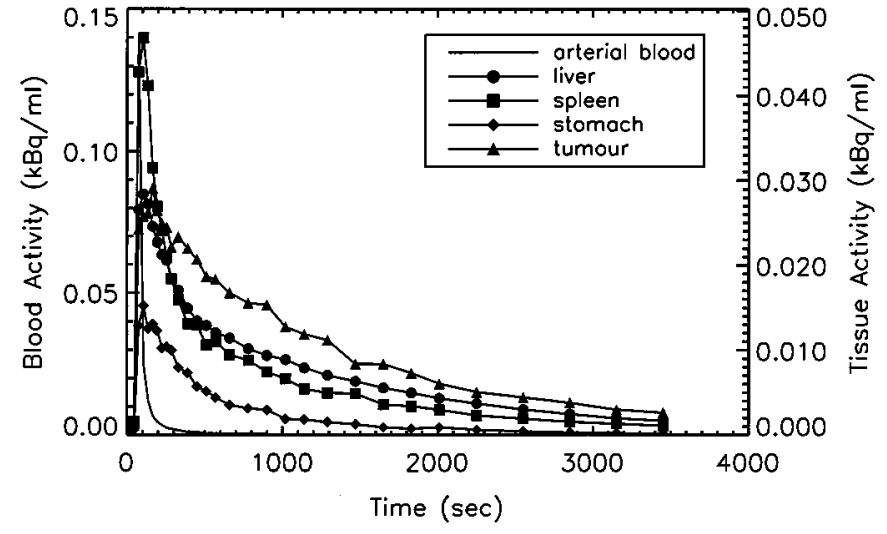

Fig. 2. Simulated noisy $2-\left[{ }^{11} \mathrm{C}\right]$ thymidine kinetics in some representative regions. A metabolite-corrected arterial blood curve which was used to simulate 2- $\left[{ }^{11} \mathrm{C}\right]$ thymidine kinetics in different tissues is also shown.

Poisson noise and blurring were added to simulate realistic sinograms. Noisy dynamic images were then reconstructed using FBP (Hann filter cutoff at the Nyquist frequency). Fig. 2 shows the metabolite-corrected arterial blood curve and noisy 2-[ $\left.{ }^{11} \mathrm{C}\right]$ thymidine kinetics in some representative tissues.

\section{Human Studies}

The clustering algorithm has been applied to a range of FDG-PET studies and three examples (two patients with brain tumor and one patient with a lung cancer) are presented in this paper. FDG-PET was chosen to assess the clustering algorithm because it is commonly used in clinical oncologic PET studies. All oncological PET studies were performed at our institution. Ethical permissions were obtained from our Institutional Review Board.

Dynamic neurologic FDG-PET studies were performed on an ECAT 951R whole-body PET tomograph (CTI/Siemens, Knoxville, TN). Throughout the study the patient's eyes were patched and ears were plugged. The patients received $400 \mathrm{MBq}$ of FDG, infused at a constant rate over a 3-min period using an automated injection pump. At least $30 \mathrm{~min}$ prior to the study, patient's hands and forearms were placed into hot water baths preheated to $44{ }^{\circ} \mathrm{C}$ to promote arterio-venous shunting. Blood samples were taken at approximately $30 \mathrm{~s}$ for the first $6 \mathrm{~min}$, and at approximately $8,10,15,30$, and $40 \mathrm{~min}$ and at the end of emission data acquisition. A dynamic sequence of 22 frames was acquired for 60 min following radiotracer administration according to the following schedule: $6 \times 10 \mathrm{~s}$, $4 \times 30 \mathrm{~s}, 1 \times 2 \mathrm{~min}, 11 \times 5 \mathrm{~min}$. Data were attenuation corrected with a post-injection transmission method [24]. Images were reconstructed on a $128 \times 128$ matrix using FBP with a Shepp and Logan filter cutoff at 0.5 of the Nyquist frequency.

The dynamic lung FDG-PET study was commenced after intravenous injection of $487 \mathrm{MBq}$ of FDG. Emission data were acquired on an ECAT 951R whole-body PET tomograph (CTI/Siemens, Knoxville, TN) over 60 min (22 frames, $6 \times 10 \mathrm{~s}, 4 \times 30 \mathrm{~s}, 1 \times 2 \mathrm{~min}$, and $11 \times 5 \mathrm{~min})$. Twenty-one arterial blood samples were taken from the pulmonary artery using a Grandjean catheter to provide an input function for kinetic modeling.

The patient details are as follows. 
Patient 1: The FDG-PET scan was done in a female patient six months after resection of a malignant primary brain tumor in the right parieto-occipital lobe. The scan was done to determine if there was evidence for tumor recurrence. A partly necrotic hypermetabolic lesion was found in the right parieto-occipital lobe that was consistent with tumor recurrence.

Patient 2: A 40-year-old woman had a glioma in the right mesial temporal lobe. The FDG-PET scan was performed at six months after tumor resection. A large hypermetabolic lesion was identified in the right mesial temporal lobe that was consistent with tumor recurrence.

Patient 3: A 67-year-old man had an aggressive mesothelioma in the left lung. In the PET images, separate foci of increased FDG uptake were seen in the contralateral lymph nodes as well as in the peripheral left lung.

As they are unnecessary for clustering and the subsequent analysis, low count areas such as the background (where the voxel values should be zero theoretically), and streaks (which are due to reconstruction errors) were excluded by zeroing voxels whose summed activity was below $5 \%$ of the mean pixel intensity of the integrated dynamic images. A $3 \times 3$ closing followed by a $3 \times 3$ erosion operation was then applied to fill any "gap" inside the intracranial/body region to which cluster analysis was applied. Parametric images of the physiological parameter $K$, which is defined as the value of $k_{1}^{*} k_{3}^{*} /\left(k_{2}^{*}+k_{3}^{*}\right)$ [25], were generated by fitting all voxels inside the intracranial/body region using Patlak graphical approach [26]. The resultant parametric images obtained for the raw dynamic images and dynamic images after cluster analysis were assessed visually. Compartmental model fitting using the three-compartment FDG model [25] was also performed on the tissue TACs extracted manually and by cluster analysis to investigate whether there is any disagreement between the parameter estimates.

\section{RESULTS}

\section{A. Validation Study}

Fig. 3 shows the segmentation results using different numbers of clusters $k$ in the clustering algorithm. The number of clusters is actually varied from 3 to 13 but only some representative samples are shown. In each of the images in Fig. 3(a)-(f), different grey levels are used to represent the cluster locations. Fig. 3 shows that when the number of clusters is small, segmentation of the data is poor. With $k=3$, the liver, marrow, and spleen merge to form a cluster and the other regions merge to form a single cluster. With $5 \leq k \leq 7$, the segmentation results improve because the blood vessels and stomach are visualized. However, the hepatic tumors are not seen and the liver and spleen are classified into the same cluster. With $k=8$, the tumors are visualized and almost all of the regions are correctly identified [Fig. 3(d)]. Increasing the value of $k$ to nine gives nearly the same segmentation as in the case of $k=8$ [Fig. 3(e)]. Further increasing the value of $k$, however, may result in poor segmentation because the actual number of tissues present in the

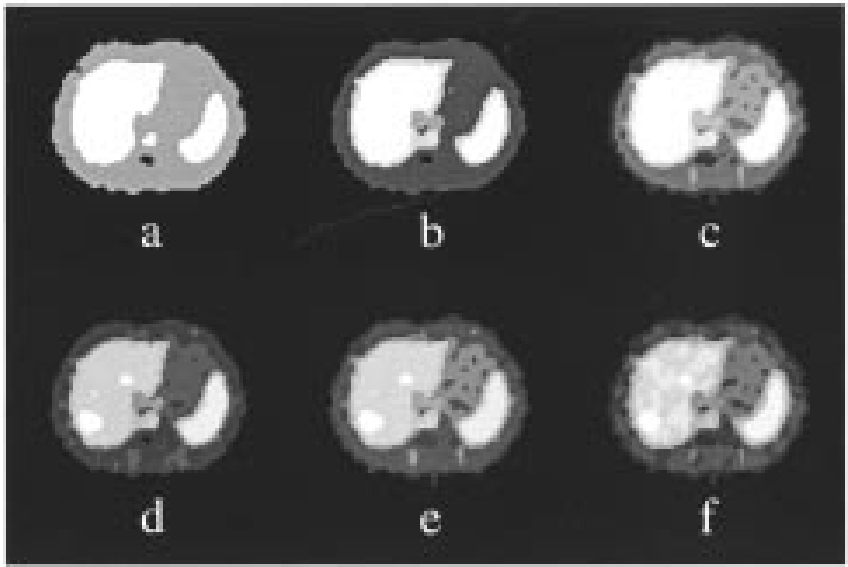

Fig. 3. Tissue segmentation obtained with different number of clusters (a) $k=3$. (b) $k=5$. (c) $k=7$. (d) $k=8$. (e) $k=9$. (f) $k=13$.

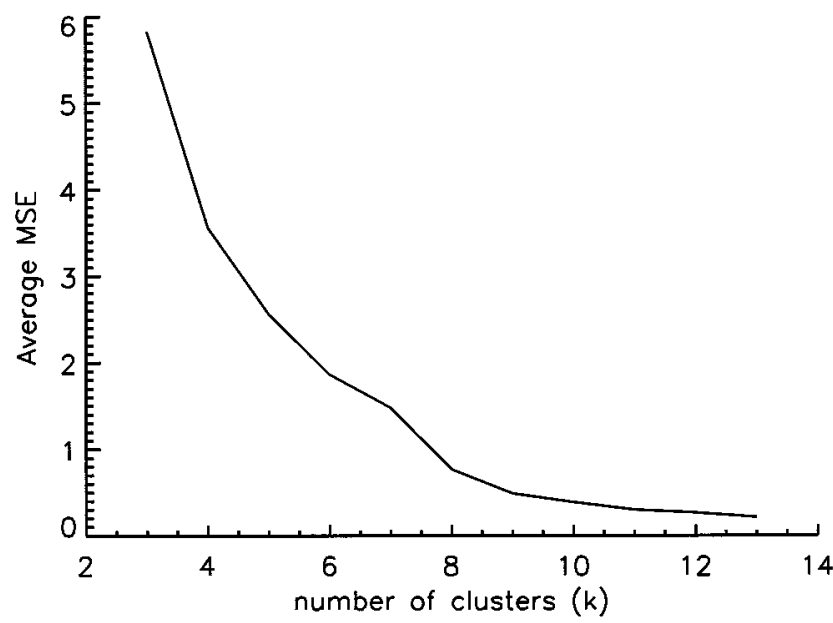

Fig. 4. Average mean squared error as a function of number of clusters.

data is less than the specified number of clusters. Homogeneous regions are therefore fragmented to satisfy the constraint on the number of clusters [Fig. 3(f)]. Thus, eight or nine clusters appear to provide reasonable segmentation of tissues in the slice and this number agrees with the various kinetics present in the data.

Fig. 4 plots the average MSE across clusters as a function of $k$. The average MSE decreases monotonically, as it drops rapidly $(k<8)$ before reaching a plateau $(k \geq 10)$. From the trend of the plot, there is no significant reduction in the average MSE with $k>12$. Furthermore, the decrease in the average MSE is nearly saturated with $k \geq 8$. These results confirm the findings of the images in Fig. 3, suggesting eight or nine as the optimal number of clusters for this dataset.

Table I tabulates the results of applying AIC and SC to determine the optimum number of clusters which is the one that gives the minimum value for the criteria. Both criteria indicate that $k=8$ is an optimal approximation to the underlying number of kinetics. It was found that a good segmentation can be achieved when the number of clusters is the same as that determined by the criteria. Conversely, the segmentation result is poor when the number of clusters is smaller than that suggested by the criteria and there is no significant improvement in segmentation when 
TABLE I

Computed Values for AIC and SC With Different Choices of the VAlue of $k$. Value ApPearing in Bold CORRESPONDS TO THE COMPUTED MINIMUM OF THE CRITERION

\begin{tabular}{lccccccccccc}
\hline & \multicolumn{10}{c}{ Number of clusters, $k$} \\
\cline { 2 - 12 } Criterion & 3 & 4 & 5 & 6 & 7 & 8 & 9 & 10 & 11 & 12 & 13 \\
\hline AIC & 99005 & 95354 & 93469 & 90904 & 88851 & $\mathbf{8 6 9 6 7}$ & 89769 & 93038 & 91994 & 90840 & 89807 \\
SC & 98654 & 94888 & 92887 & 90206 & 88038 & $\mathbf{8 6 0 3 8}$ & 88725 & 91878 & 90719 & 89450 & 88301 \\
\hline
\end{tabular}

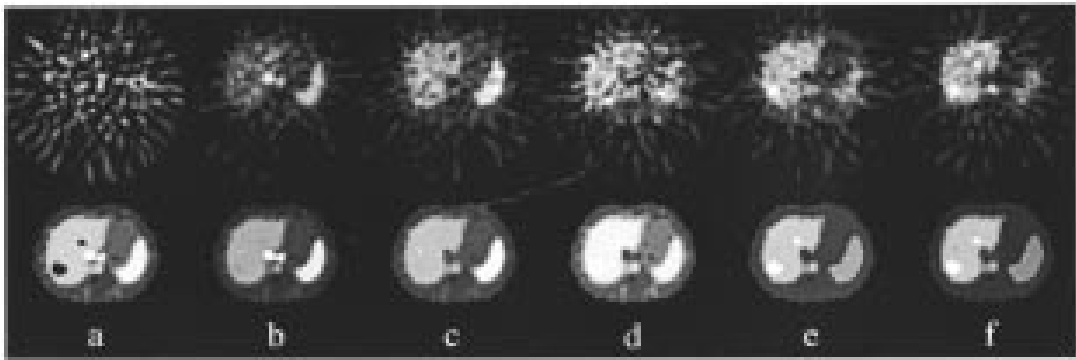

Fig. 5. Single slice of simulated 2-[11 C]thymidine PET study. Top row shows the original reconstructed images at (a) 15-s postinjection. (b) 75-s postinjection. (c) 135-s postinjection. (d) 285-s postinjection. (e) 1020-s postinjection. (f) 2850-s postinjection. Bottom row shows the same slice at identical time points after cluster analysis. Individual images are scaled to their own maximum.

the number of clusters is larger than that determined by the criteria. The heuristic information given by both criteria also support our visual interpretation of the clustering results, suggesting that the criteria are reasonable approaches to objectively determine the number of clusters.

Application of the clustering algorithm to the simulated PET data is shown in Fig. 5. The number of clusters is eight, corresponding to the optimum number of clusters determined by the statistical criteria. The SNR of the images is markedly improved after clustering. In addition, the blood vessels are clearly seen in the frame sampled at 15 and $75 \mathrm{~s}$ after clustering but not in the corresponding frame in the original data. In the original images, it is difficult to identify different tissues which may be due to reconstruction effects and inhomogeneous noise. However, the liver, spleen, muscle, marrow, stomach, and tumors, are clearly delineated by the clustering algorithm (bottom row of the figure).

\section{B. Human Studies}

Segmentation results are shown for dynamic neurologic (Fig. 6) and lung (Fig. 7) FDG-PET studies. The clusters are represented by differing grey scales and slices were sampled at the level where the lesions were seen on the original reconstructed data. Since there is no a priori knowledge about the optimum number of clusters, the value of $k$ was varied in order to determine the optimal segmentation using the AIC and $\mathrm{SC}$ as in the phantom study. For Fig. 6, eight clusters were found to give the optimal segmentation for these data sets. The locations of the tumors and the rim of increased glucose uptake are identified correctly by the clustering algorithm with the optimal value of clusters.

For Fig. 7, the number of clusters was varied from 3 to 13 and only some representative results are shown. Similar to the simulation study, the segmentation results are poor when the number of clusters is small $(k=3)$, while the segmentation is gradually improved by increasing the number of clusters. Based on the

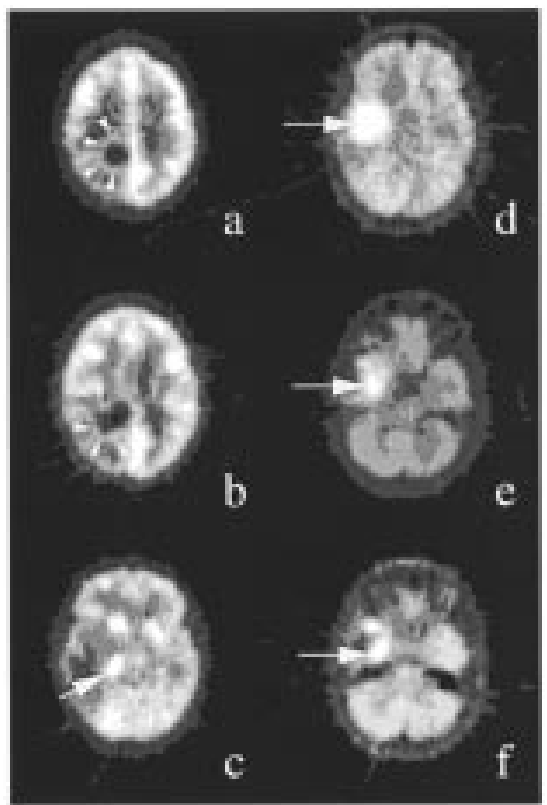

Fig. 6. Tissue segmentation obtained from Patient 1 at (a) slice 10; (b) slice 13; and (c) slice 21 and Patient 2 at (d) slice 21; (e) slice 24; and (f) slice 26. The number of clusters used is eight. The locations of the solid hypermetabolic portions of the tumors (arrows) and the small rim of increased glucose uptake (arrow heads) identified by cluster analysis are shown.

AIC and SC, the optimum numbers of clusters for the selected slices $(4,19$, and 24$)$ were found to be 8,8 , and 9 , respectively. It is not surprising that the optimum number of clusters is different for different slices because of the differing number of anatomical structures contained in the plane and the heterogeneity of tracer uptake in tissues. Nevertheless, the tumor (slice 4), right lung and muscle (slices 4, 19, and 24), blood pool (slices 4, 19, and 24), separate foci of increased FDG uptake (slices 19 and 24), and the injection site (slices 4, 19, and 24) are identifiable with the optimum number of clusters. 


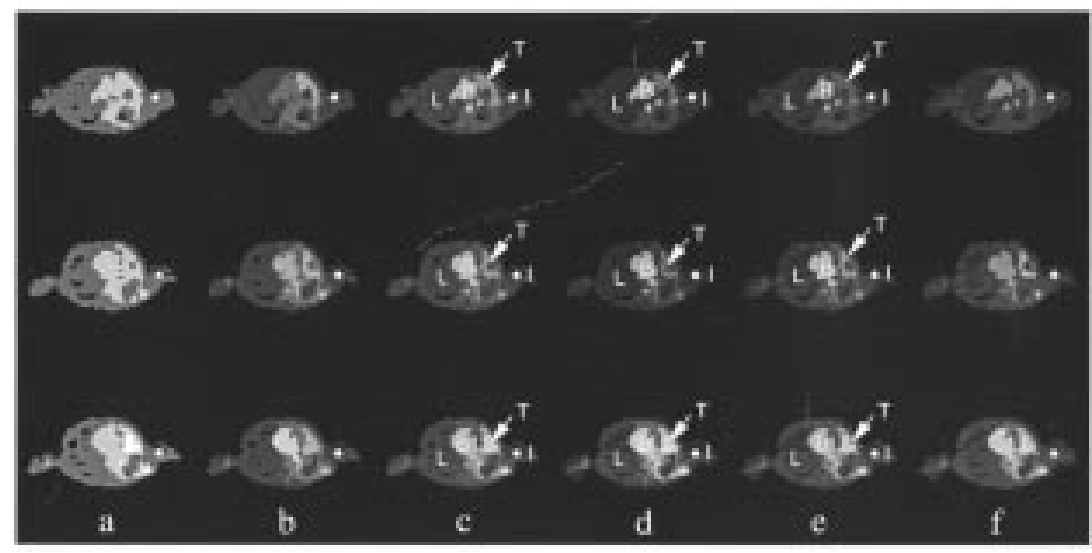

Fig. 7. Tissue segmentation of the dynamic lung FDG-PET data from Patient 3 in three selected slices-4 (top row), 19 (middle row) and 24 (bottom row) with different number of clusters: (a) $k=4$; (b) $k=7$; (c) $k=8$; (d) $k=9$; (e) $k=10$; (f) $k=12$. (I = injection site; $\mathrm{B}=$ blood pool; $\mathrm{L}=1$ lung; $\mathrm{T}=$ tumor).

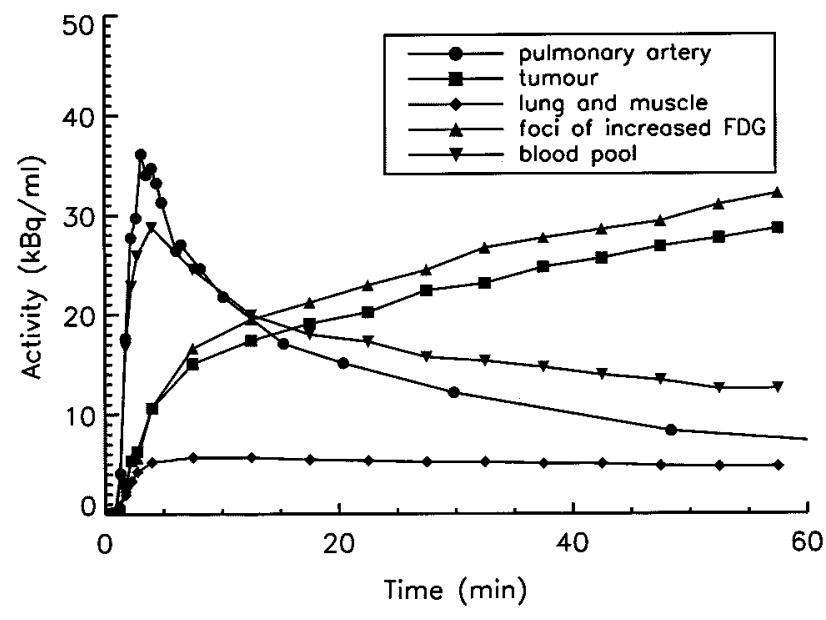

Fig. 8. Extracted tissue TACs corresponding to the tumor, lung, and muscle, foci of increased FDG uptake, and blood pool. The measured blood TAC at the pulmonary artery is also shown.

TABLE II

COMPARTMENTAL MODELLING OF THE TUMOR TACS OBTAINED By MaNUally ROI DELINEATION AND BY CluSTER ANALYSIS. VALUES ARE GIVEN AS Estimate $\pm \%$ CV

\begin{tabular}{lll}
\hline Parameter & Manual delineation & Cluster analysis \\
\hline$k_{1}^{*}(\mathrm{ml} / \mathrm{min} / \mathrm{g})$ & $0.854 \pm 17.1$ & $0.921 \pm 18.2$ \\
$k_{2}^{*}\left(\mathrm{~min}^{-1}\right)$ & $1.987 \pm 21.2$ & $2.096 \pm 22.5$ \\
$k_{3}^{*}\left(\mathrm{~min}^{-1}\right)$ & $0.099 \pm 0.9$ & $0.100 \pm 0.9$ \\
$k_{4}^{*}\left(\mathrm{~min}^{-1}\right)$ & $0.018 \pm 1.1$ & $0.017 \pm 1.2$ \\
$K(\mathrm{ml} / \mathrm{min} / \mathrm{g})$ & $0.041 \pm 5.3$ & $0.042 \pm 5.3$ \\
\hline
\end{tabular}

Fig. 8 shows the measured blood TAC at the pulmonary artery and the extracted tissue TACs for the tumor (from slice 4), lung and muscle (from slice 19), foci of increased FDG uptake (from slice 24), and the blood pool (from slice 19) using the corresponding optimal value of clusters.

The extracted tissue TACs obtained by cluster analysis and manual ROI delineation were fitted to the three-compartment FDG model using nonlinear least-squares method and the results obtained for the tumor tissue TAC (Patient 2) are summarized in Table II. There was a close agreement between the

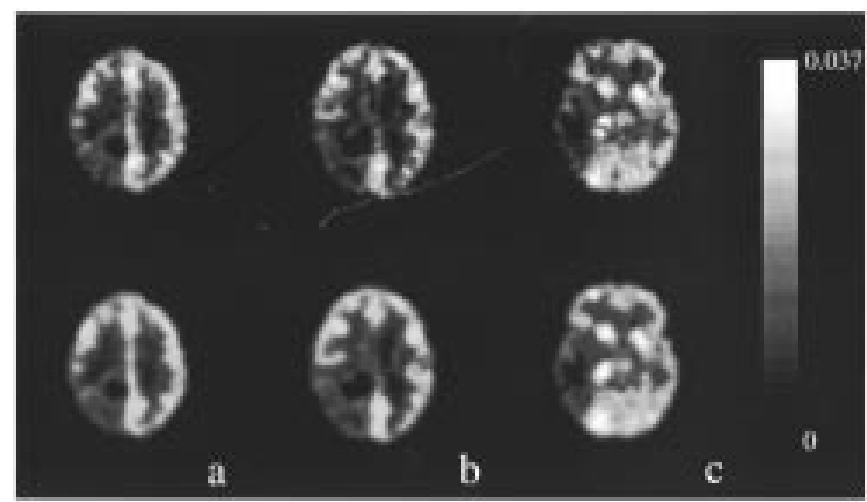

Fig. 9. Parametric images on a pixel-by-pixel basis of $K$ obtained from Patient 1: (a) slice 10; (b) slice 13; (c) slice 21. Top row shows the images obtained from the raw dynamic images and bottom row shows the images obtained from dynamic images after cluster analysis. The images have been smoothed slightly for better visualization.

parameter estimates for the tissue TACs obtained by different methods in terms of the estimate and the coefficient of variation $(\mathrm{CV})$, which is defined as the ratio of the standard deviation of the parameter estimate to the value of the estimate. Similar results were also found for other regions.

Fig. 9 shows the parametric images of physiological parameters $K$ obtained from the neurologic study for Patient 1 in the three selected slices. The top and bottom rows of the images correspond to the results obtained from pixel-by-pixel fitting the TACs in the raw dynamic PET data and data after cluster analysis, respectively. The $K$ images are relatively noisy when compared to the data after cluster analysis because of the high noise levels of PET data which hampered reliable parametric image generation. However, the visual quality of the $K$ images improves markedly with cluster analysis due to the increased SNR of the dynamic images. Low-pass filtering of the original parametric images may improve the SNR but clustering should produce better results because it takes the tissue TACs with similar temporal characteristics for averaging. Meanwhile, low-pass filtering only makes use of the spatial (adjacent pixels) information for filtering and this will only further degrade the spatial resolution. 


\section{DISCUSSION}

We have described a tissue segmentation scheme for dynamic PET data using cluster analysis. The proposed scheme is an attempt to overcome some of the limitations associated with conventional ROI analysis. It is able to provide statistically meaningful clusters because the entire sequence of images are analyzed and different kinetic behaviors and the associated regions are extracted from the data set, as long as there is a finite number of kinetics in the data. Once the segmentation process is finished, the extracted TACs, i.e., the cluster centroids, are then mapped back to the original data space for all voxels. Thus, an improved SNR can be achieved because each voxel in the mapped data space is represented by one of the cluster centroids each of which possesses a higher statistical significance than an individual TAC in the same spatial location. Therefore, the extracted TACs obtained by cluster analysis should be more consistent and reproducible.

It is difficult to identify obvious cluster centroids in PET data because they are multidimensional and noisy. Therefore, initial centroids are needed for the proposed algorithm. The initial cluster centroids do not have to be accurate because they are only used as seeds to start the algorithm. However, if the starting centroids are far from the final cluster centroids, more iterations may be required. An incorrect initial selection may occur if a noisy outlier is chosen, resulting in a cluster with a single member. For this case, a lower bound on the final number of members in a cluster should be incorporated to prevent the cluster from being exhausted.

The optimum number of clusters for cluster analysis is usually not known a priori. The number of clusters, $k$, is also dependent on a number of factors mentioned previously. In addition, different choices for the values of $k$ may result in different partitions of data. In this study, we limited the range for the values of $k$ and applied the clustering algorithm to the simulated and real data. Nevertheless, it is reasonable to assume that the limited number of clusters used in this study is feasible, given that there is a finite number of kinetics present in the data. With the use of information-theoretic approaches to cluster validation, one can objectively determine the optimum number of clusters for the given dataset. However, caution should be taken when using the criteria as they are model dependent. The optimum number of clusters suggested by the criteria may not make sense if the specified probability distribution function for the observed data is not appropriate. There are a number of statistical criteria for the determination of the optimal number of clusters in addition to those used in this study and we are currently exploring various approaches to the cluster validation problem.

A limitation of the proposed algorithm is that it cannot differentiate anatomical structures which are unconnected but have similar kinetics. Additional information such as the geometry and the coordinates of the structure concerned are required. Another related issue is tissue heterogeneity [27] although this effect is usually ignored. In this study, we did not attempt to solve this problem for cluster analysis. However, some heuristic interpretations could be made. In anatomy, most of the anatomical structures are discrete and well separated, they should easily be segmented by the proposed algorithm. Partial volume ef- fects of the PET system cause these structures to overlap with kinetics which are a mixture of the structures involved. As a finite number of clusters is assumed to be present in the raw PET data, the clustering algorithm will automatically look for the cluster centers that best represent the data set without any $a$ priori knowledge about the data and without violating the specified number of clusters. Therefore, certain regions which are indeterminate but their kinetics are similar, may be grouped together due to the constraint on the number of clusters, resulting in the formation of vague clusters. Further studies are required to investigate tissue heterogeneity in cluster analysis.

In the clustering process, low count areas were excluded by simple thresholding. To obtain better results, one can coregister the PET image frames with high spatial resolution MR images, and generate a mask for PET images to which cluster analysis is applied, although caution in regard to ROI selection should be used. On the other hand, good clustering results are expected if there is less noise in the PET data. This may be achieved by reconstructing images using statistical reconstruction methods.

Patient motion may also affect the accuracy of quantification and clinical interpretation. Motion may be interpreted as activity changes, causing the clustering algorithm to take these "false kinetics" into account, resulting in incorrect segmentation. It is desirable that this effect is eliminated by means of some motion correction scheme [28], [29].

In earlier work, O'Sullivan [15] used cluster analysis as an intermediate step to extract "homogeneous" TACs from data containing a heterogeneous mix of kinetics resulting from spillover and partial volume effects for parametric mapping. However, in this current work, we used cluster analysis to extract kinetic data with different temporal characteristics as well as for parametric mapping. This is important for data analysis because data with different temporal behavior are better characterized by the extracted features seen in a spatial map. A spatial map is simpler to interpret when compared to the original multidimensional data. However, similar to O'Sullivan's approach [15], our method is data driven and is independent of the properties of tracer that may be required by other methods [17]. Thus, our approach can be applied to a wide range of tracer studies.

The clustering process usually converges quite rapidly. Convergence can typically be reached within five to ten iterations, which takes approximately $5 \mathrm{~s}$ in total on a UltraSPARC- 1 workstation (143-MHz CPU, $64 \mathrm{MB}$ of memory) to search for eight clusters in a single slice $(128 \times 128$ voxels $)$ of 22 frames with randomised initial cluster centroids.

Fast generation of parametric images is now possible with current high-speed computer workstations. However, overestimation of parameters and negative parameter estimates, which are not physiologically feasible, occur often when the data are too noisy. Reliable parametric imaging is, therefore, largely dependent on the noise levels inherent in the data which affect, in addition to meaningful parameter estimation, the time required to converge as well as the convergence. Our clustering algorithm may be useful as a preprocessing step before fast generation of parametric images since only a few characteristic curves, which have high statistical significance, need to be fitted as compared to conventional pixel-by-pixel parametric image generation where many thousands of very noisy tissue TACs must 
be analyzed. The computational advantage and time savings for generation of parametric images (fitting many thousands of kinetic curves versus several curves) are apparent.

One of the potential applications of our clustering algorithm is in noninvasive quantitative PET. We have proposed a simultaneous estimation approach to estimate the input function and physiological parameters simultaneously with two or more ROIs and our results with in vivo PET data are promising [18]. The method is still limited, however, by the selection of ROIs whose TACs must have distinct kinetics. As the ROIs are drawn manually on the PET images, reproducibility is difficult to achieve. The feasibility of using the kinetic curves extracted by cluster analysis for noninvasive quantification of physiological parameters has been investigated and some preliminary data have been reported [30]. Thus, cluster analysis may be useful as a preprocessing step before our noninvasive modeling technique.

\section{CONCLUSION}

We present an approach to automatically segment tissues in dynamic PET images using cluster analysis. Our preliminary data from a simulated PET study indicate that accurate tissue segmentation can be achieved and may replace manual ROI delineation. This approach may be potentially useful as a preprocessing step before noninvasive image-based modeling and fast generation of parametric images. Our results have encouraged us to investigate the applicability of this approach to whole-body PET for lesion localization and assessment of treatment response in a variety of oncological conditions.

\section{REFERENCES}

[1] J. C. Mazziotta, C. A. Pelizzari, G. T. Chen, F. L. Bookstein, and D. Valentino, "Region of interest issues: The relationship between structure and function in the brain," J. Cereb. Blood Flow Metab., vol. 11, pp. A51-A56, 1991.

[2] E. J. Hoffman, S. C. Huang, and M. E. Phelps, "Quantitation in positron emission computed tomography: 1. Effect of object size," J. Comput. Assist. Tomog., vol. 3, pp. 299-308, 1979.

[3] C. C. Meltzer, J. P. Leal, H. S. Mayberg, H. N. Wagner, and J. J. Frost, "Correction of PET data for partial volume effects in human cerebral cortex by MR imaging," J. Comput. Assist. Tomog., vol. 14, pp. 561-570, 1990.

[4] H. W. Müller-Gärtner, J. M. Links, J. L. Price, R. N. Bryan, E. McVeigh, J. P. Leal, C. Davatzikos, and J. J. Frost, "Measurement of radiotracer concentration in brain gray matter using positron emission tomography: MRI-based correction for partial volume effects," J. Cereb. Blood Flow Metab., vol. 12, pp. 571-583, 1992.

[5] J. B. A. Maintz and M. A. Viergever, "A survey of medical image registration," Med. Anal., vol. 2, pp. 1-37, 1998.

[6] P. T. Fox, J. S. Perlmutter, and M. E. Raichle, "A stereotatic method of anatomical localization for positron emission tomography," J. Comput. Assist. Tomog., vol. 9, pp. 141-153, 1985.

[7] J. Talairach, P. Tournoux, and M. Rayport, Co-Planar Stereotaxic Atlas of the Human Brain. New York: Thieme, 1988.
[8] P. Thompson and A. Toga, "A surface-based technique for warping three-dimensional images of the brain," IEEE Trans. Med. Imaging, vol. 15, pp. 402-417, Aug. 1996.

[9] M. Bomans, K. H. Hohne, U. Tiede, and M. Riemer, "Three-dimensional segmentation of MR images of the head for 3-D display," IEEE Trans. Med. Imaging, vol. 9, pp. 177-183, June 1990.

[10] Z. Liang, J. R. MacFall, and D. P. Harrington, "Parameter estimation and tissue segmentation from multispectral MR images," IEEE Trans. Med. Imaging, vol. 13, pp. 441-449, Sept. 1994.

[11] W. M. Wells III, W. E. L. Grimson, R. Kikinis, and F. A. Jolesz, "Adaptive segmentation of MRI data," IEEE Trans. Med. Imaging, vol. 15, pp. 429-442, Aug. 1996

[12] C. Chatfield and A. J. Collins, Introduction to Multivariate Analysis. New York: Chapman \& Hall, 1980.

[13] C. Goutte, P. Toft, E. Rostrup, F. Å. Nielsen, and L. K. Hansen, "On clustering fMRI time series," NeuroImage, vol. 9, pp. 298-310, 1999.

[14] P. Filzmoser, R. Baumgartner, and E. Moser, "A hierarchical clustering method for analyzing functional MR images," Magn. Resonance Imaging, vol. 17, pp. 817-826, 1999.

[15] F. O' Sullivan, "Imaging radiotracer model parameters in PET: A mixture analysis approach," IEEE Trans. Med. Imaging, vol. 12, pp. $399-412$, Sept. 1993.

[16] J. Ashburner, J. Haslam, C. Taylor, V. J. Cunningham, and T. Jones, "A cluster analysis approach for the characterization of dynamic PET data," in Quantification of Brain Function using PET, R. Myers, V. Cunningham, D. Bailey, and T. Jones, Eds. San Diego, CA: Academic, 1996, pp. 301-306.

[17] Y. Kimura, H. Hsu, H. Toyama, M. Senda, and N. M. Alpert, "Improved signal-to-noise ratio in parametric images by cluster analysis," $\mathrm{Neu}$ rolmage, vol. 9, pp. 554-561, 1999.

[18] K. P. Wong, D. Feng, S. R. Meikle, and M. J. Fulham, "Simultaneous estimation of physiological parameters and the input function: In vivo PET data," IEEE Trans. Inform. Technol. Biomed., vol. 5, pp. 67-76, Mar. 2001.

[19] I. G. Zubal, C. R. Harrell, E. O. Smith, Z. Rattner, G. Gindi, and P. B. Hoffer, "Computerized three-dimensional segmented human anatomy," Med. Phys., vol. 21, pp. 299-302, 1994.

[20] L. Cooper, "M-dimensional location models: Application to cluster analysis," J. Reg. Sci., vol. 13, pp. 41-54, 1973.

[21] H. Akaike, "A new look at the statistical model identification," IEEE Trans. Automat. Contr., vol. AC-19, pp. 716-723, Dec. 1974.

[22] G. Schwarz, "Estimating the dimension of a model," Ann. Stat., vol. 6, pp. 461-464, 1978.

[23] V. J. Cunningham and T. Jones, "Spectral analysis of dynamic PET studies," J. Cereb. Blood Flow Metab., vol. 13, pp. 15-23, 1993.

[24] P. K. Hooper, S. R. Meikle, S. Eberl, and M. J. Fulham, "Validation of post injection transmission measurements for attenuation correction in neurologic FDG PET studies," J. Nucl. Med., vol. 37, pp. 128-136, 1996.

[25] S. C. Huang, M. E. Phelps, E. J. Hoffman, K. Sideris, C. Selin, and D. E. Kuhl, "Noninvasive determination of local cerebral metabolic rate of glucose in man," Amer. J. Physiol., vol. 238, pp. E69-E82, 1980.

[26] C. S. Patlak, R. G. Blasberg, and J. Fenstermacher, "Graphical evaluation of blood-to-brain transfer constants from multiple-time uptake data," $J$ Cereb. Blood Flow Metab., vol. 3, pp. 1-7, 1983.

[27] K. Schmidt, G. Lucignani, R. M. Moresco, G. Rizzo, M. C. Gilardi, C. Messa, F. Colombo, F. Fazio, and L. Sokoloff, "Errors introduced by tissue heterogeneity in estimation of local cerebral glucose utilization with current kinetic models of the $\left[{ }^{18} \mathrm{~F}\right]$ fluorodeoxyglucose method," $J$. Cereb. Blood Flow Metab., vol. 12, pp. 823-834, 1992.

[28] M. Menke, M. S. Atkins, and K. R. Buckley, "Compensation methods for head motion detected during PET imaging," IEEE Trans. Nucl. Sci., vol. 43, pp. 310-317, Feb. 1996.

[29] R. R. Fulton, S. R. Meikle, S. Eberl, J. Pfeiffer, and M. J. Fulham, "Correction for head movements in positron emission tomography using an optical motion tracking system," presented at the 2000 IEEE Medical Imaging Conf., Lyon, France, Oct. 2000, pp. 17/58-17/62.

[30] K. P. Wong, D. Feng, S. R. Meikle, and M. J. Fulham, "Non-invasive determination of the input function in PET by a Monte Carlo approach and cluster analysis," J. Nucl. Med., vol. 62, p. 183P, 2001. 